\title{
HILBERT CURVES OF QUADRIC FIBRATIONS
}

\author{
ANTONIO LANTERI
}

\begin{abstract}
Let $(X, L)$ be a quadric fibration over a smooth curve. The explicit equation of the corresponding Hilbert curve $\Gamma$ is obtained. The geometry of $\Gamma$ reflects some structure properties of $(X, L)$; in particular, its special shape allows us to recognize that $(X, L)$ is a quadric fibration. In fact $\Gamma$ is reducible into $\operatorname{dim} X-2$ parallel lines with prescribed slope, evenly spaced, plus a conic. On the other hand, this conic can itself be regarded as the Hilbert curve of a polarized surface only in very rare circumstances.
\end{abstract}

2010 Mathematics Subject Classification: 14C20, 14N30, 14M99, $14 \mathrm{H} 50$.

Keywords: polarized manifold, Hilbert curve, quadric fibration, projective bundle.

\section{INTRODUCTION}

Let $(X, L)$ be a polarized manifold of dimension $n$ and assume that the canonical bundle $K_{X}$ is not numerically trivial. Then one can associate to $(X, L)$ a plane algebraic curve $\Gamma:=\Gamma_{(X, L)}$ of degree $n$, called the Hilbert curve of $(X, L)$. Let $x, y$ be complex variables and consider the polynomial $p \in \mathbb{C}[x, y]$ (in fact $p \in \mathbb{Q}[x, y]$ ), whose restriction to the integral lattice $\mathbb{Z}^{2} \subset \mathbb{C}^{2}$ is the polynomial expression provided by the Riemann-Roch theorem for the Euler-Poincaré characteristic $\chi\left(x K_{X}+y L\right)$. Then $\Gamma$ is the complex affine curve defined by $p(x, y)=0$. As shown in [3], $\Gamma$ reflects interesting properties of the pair $(X, L)$. In particular, if the nef value of $(X, L)$ is $\tau:=a / b$ (with $a, b$ relatively prime positive integers), then $\Gamma$ is reducible and contains $a-1$ parallel lines as components [3, Theorem 6.1].

Inspired by the study of the Hilbert curve of a projective bundle [9], in this paper we focus on the case in which $(X, L)$ is a quadric fibration over a smooth curve, and we determine the explicit expression of the polynomial $p$ defining the corresponding Hilbert curve $\Gamma$ (Section 1 ). It turns out that $\Gamma$ consists of a conic $G$ in the $(x, y)$-plane with center at $C=\left(\frac{1}{2}, 0\right)$ plus $n-2$ parallel lines $\ell_{1}, \ldots, \ell_{n-2}$ with slope $n-1$, evenly spaced (that is, the distance of each line from the next is always the same), and giving rise to a symmetric configuration with respect to $C$. We show that the conic $G$ is reducible into two lines $\ell$ and $\lambda$ if and only if the quadric fibration has no singular fibers. Moreover, in this case one of these two lines, say $\ell$, has slope $n-1$. In particular, if $n$ is odd, then $\ell$ coincides with $\ell_{1}$, up to renaming, hence $\Gamma$ is even non-reduced. This generalizes [3, Proposition 4.8].

Assuming that the classes of $K_{X}$ and $L$ in $\operatorname{Num}(X)$ are linearly independent, and $K_{X}+(n-1) L$ is nef, we prove that the equation we obtained for $\Gamma$ characterizes the fact that $(X, L)$ is a quadric fibration over a smooth curve (Section 2). This is the main result in the paper and can be regarded as an analogue for $\mathbb{Q}$-fibrations of what was already proved in [9, Theorem 4.1] for scrolls over a smooth curve.

A further natural question, stimulated by [3, Problem $6.6(1)]$, is concerned with the conic $G$. Clearly, $G$ is invariant under the involution $(x, y) \mapsto(1-x, y)$ induced 
A. LANTERI

by Serre duality. A non-obvious question, however, is whether $G$ can itself be regarded as the Hilbert curve of some polarized surface. Even confining to quadric fibrations with no singular fibers, we show that if $n \geq 3$ the answer is positive only for $n=3$ (Section 3). On the other hand, for $n=2$, in which case $\Gamma=G$, we analyze whether $\Gamma$ can occur as the Hilbert curve of other polarized surfaces, distinct from the conic bundle $(X, L)$, providing a complete answer to this question (Section 4).

\section{Notation AND TERMinOLOGY}

Varieties considered in this paper are defined over the field $\mathbb{C}$ of complex numbers. We use the standard notation and terminology from algebraic geometry. A manifold is any smooth projective variety. Tensor products of line bundles are denoted additively. The pullback of a vector bundle $\mathcal{F}$ on a manifold $X$ by an embedding $Y \hookrightarrow X$ is simply denoted by $\mathcal{F}_{Y}$. We denote by $K_{X}$ the canonical bundle of a manifold $X$. We use the symbol $\equiv$ to denote numerical equivalence. A polarized manifold is a pair $(X, L)$ consisting of a manifold $X$ and an ample line bundle $L$ on $X$. The word scroll has to be intended in the classical sense, i. e., a polarized manifold $(X, L)$ such that $X$ is a $\mathbb{P}^{k}$-bundle over a variety $Y$ and $L_{F}=\mathcal{O}_{\mathbb{P}^{k}}(1)$ for any fiber $F \cong \mathbb{P}^{k}$.

\section{(0.1) Quadric fibrations}

As in [5], we say that a polarized manifold $(X, L)$ of dimension $n \geq 2$ is a quadric fibration (a conic fibration if $n=2$ ) over a curve if there exists a surjective morphism $\pi: X \rightarrow B$ onto a smooth curve $B$ such that any general fiber $F$ of $\pi$ is a smooth quadric hypersurface $\mathbb{Q}^{n-1} \subset \mathbb{P}^{n}$ of the complex projective space $\mathbb{P}^{n}$, and $L_{F}=\mathcal{O}_{\mathbb{Q}^{n-1}}(1)$.

We point out that this definition (the classical one) is slightly more general than that frequently adopted in adjunction theory [2, p. 81]. Actually, since $K_{X}+(n-1) L$ restricts trivially to the fibers of $\pi$, we have that $K_{X}+(n-1) L=\pi^{*} \mathcal{A}$ for some line bundle $\mathcal{A}$ on $B$, not necessarily ample. Let $a:=\operatorname{deg} \mathcal{A}$. There are three possibilities, namely:

i) $a<0$,

ii) $a=0$,

iii) $a>0$.

Clearly $X \not \mathbb{P}^{n}$, since $X$ has Picard number $\rho \geq 2$, hence $K_{X}+n L$ is nef $[6$, Theorem 11.2]. In case i) $K_{X}+(n-1) L$ is not nef, and then [6, Theorem 11.7] implies that $(X, L)$ is a scroll (and a quadric fibration at the same time). This immediately gives $n=2$. Thus $p: X \rightarrow C$ is a $\mathbb{P}^{1}$-bundle over a smooth curve $C$; moreover, $C=\mathbb{P}^{1}$ by the Riemann-Hurwitz theorem; hence $X$ is a SegreHirzebruch surface $\mathbb{F}_{e}$, and the fact that it is endowed with two distinct fibrations $\left(p\right.$ and $\pi$ ) implies $e=0$, i.e., $X=\mathbb{P}^{1} \times \mathbb{P}^{1}$. Then $L=\mathcal{O}(2,1)$ since the fibers of $\pi$ and of $p$ have degree 2 and 1 with respect to $L$, respectively. Therefore $(X, L)=\left(\mathbb{P}^{1} \times \mathbb{P}^{1}, \mathcal{O}(2,1)\right)$. In case ii) $K_{X}+(n-1) L$ is numerically trivial. Let us point out the following fact.

Lemma 1. Let $(X, L)$ be an $n$-dimensional quadric fibration over B. The following are equivalent:

1) $\operatorname{rk}\left\langle K_{X}, L\right\rangle=1$ (i.e. $(X, L)$ is as in the degenerate case from the point of view of the Hilbert curve (see (0.2)); 
2) $K_{X}+(n-1) L$ is numerically trivial;

3) Either $n=2$, in which case $X$ is any del Pezzo surface except $\mathbb{P}^{2}$ and $L=-K_{X}$, or $n=3$ and $(X, L)=\left(\mathbb{P}^{1} \times \mathbb{P}^{1} \times \mathbb{P}^{1}, \mathcal{O}(1,1,1)\right)$.

Proof. The fact that 1) implies 2) is a simple computation. Actually, suppose that $a \neq 0$ (otherwise 2) is obvious), and let $\lambda K_{X}+\mu L=0$ in $\operatorname{Num}(X)$ for some integers $\lambda, \mu$ not both zero. Note that $\pi^{*} \mathcal{A} \equiv a F$, where $F$ is a fiber of $\pi$. By replacing the expression of $K_{X}$ in the equality we get

$$
0=\lambda((1-n) L+a F)+\mu L=\lambda(\mu+1-n) L+a F,
$$

which gives a contradiction, since the classes of $L$ and $F$ are linearly independent. To prove that 2) implies 3), observe that $-K_{X}$ is ample, hence $X$ is a Fano manifold. As such, $X$ is simply connected [4, Corollary 5.7], hence $\operatorname{Pic}(X)$ is torsion free, and therefore $(X, L)$ is a del Pezzo manifold. Then the assertion is obvious for $n=2$ while it follows from Fujita's classification [5, Theorem 8.11, p. 72] for $n \geq 3$. Actually, recall that $\operatorname{Pic}(X) \cong \mathbb{Z}$ if $n \geq 3$ and $(X, L)$ has degree $d \leq 5$ or $d=8$. Thus, $6 \leq d \leq 7$, but a direct check shows that the only one admitting a $\mathbb{Q}$-fibration structure among those del Pezzo manifolds, is the pair in 3) (e.g., see [8, Theorem]. The fact that 3) implies 1) is obvious.

Finally, in case iii) $\mathcal{A}$ is ample, i. e., $(X, L)$ is a quadric fibration in the adjunction theoretic sense $[2$, p. 81$]$. The above discussion is summarized by the following

Proposition 2. Let $(X, L)$ be a quadric fibration over a smooth curve $B$. Then one of the following facts holds:

(i) $(X, L)=\left(\mathbb{P}^{1} \times \mathbb{P}^{1}, \mathcal{O}(2,1)\right)$;

(ii) $K_{X}+(n-1) L$ is numerically trivial;

(iii) $(X, L)$ is a quadric fibration in the adjunction theoretic sense.

The enumeration agrees with that used at the beginning of (0.1). In particular, this clarifies the relation between the notion of classical quadric fibration and the adjunction theoretic one. Now let $(X, L)$ be a quadric fibration over $B$. We know that every fiber of $\pi$ is reduced for any $n \geq 2$, and irreducible if $n \geq 3$. Moreover, we claim that

(*) singular fibers, if any, are quadric cones with an isolated singular point and $L$ induces the hyperplane bundle on each of them.

In case i) $(X, L)$ has no singular fibers hence there is nothing to prove. We can thus assume that $K_{X}+(n-1) L$ is nef, and then assertion $(*)$ follows from [6, (11.8.5), argument in (5-ii) at pp. 100-101]. In particular, for $n=2$, any singular fiber has the form $e_{1}+e_{2}$, where $e_{1}, e_{2}$ are two distinct $(-1)$-curves in $X$ with $e_{i} L=e_{1} e_{2}=1$.

A crucial fact concerning quadric fibrations is that $X$ can be embedded fiberwise into a projective bundle over $B$ (see e.g. [5, Section 4]). Actually, $L$ embeds every fiber $F_{u}=\pi^{-1}(u), u \in B$, of $\pi$ as a quadric hypersurface in $\mathbb{P}^{n}$, hence $h^{0}\left(L_{F_{u}}\right)=n+1$. Therefore $\mathcal{E}:=\pi_{*} L$ is a vector bundle of rank $n+1$ on $B$. Set $P:=\mathbb{P}(\mathcal{E})$, let $\xi$ be the tautological line bundle on $P$ and let $\tilde{\pi}: P \rightarrow B$ be the projection. Then $X$ embeds fiberwise into $P$, i.e., $\left.\tilde{\pi}\right|_{X}=\pi$; moreover, $\xi_{X}=L$, and $X$ can be regarded as a divisor in the linear system $\left|2 \xi-\tilde{\pi}^{*} \mathcal{B}\right|$ for some line bundle $\mathcal{B}$ on $B$. We have $K_{P}=-(n+1) \xi+\widetilde{\pi}^{*}\left(K_{B}+\operatorname{det} \mathcal{E}\right)$ by the canonical bundle 
formula, hence, by adjunction,

$$
K_{X}=\left(K_{P}+X\right)_{X}=-(n-1) L+\pi^{*}\left(K_{B}+\operatorname{det} \mathcal{E}-\mathcal{B}\right) .
$$

This identifies the line bundle $\mathcal{A}$ on $B$ such that $K_{X}+(n-1) L=\pi^{*} \mathcal{A}$, as $\mathcal{A}=$ $K_{B}+\operatorname{det} \mathcal{E}-\mathcal{B}$. Let $q$ be the genus of the base curve $B$ and set

$$
c:=2 q-2, \quad e:=\operatorname{deg} \mathcal{E}, \quad b:=\operatorname{deg} \mathcal{B} .
$$

Then,

$$
a=c+e-b \geq 0,
$$

except in case i), according to Proposition 2. Moreover, equality holds exactly for the pairs listed in Lemma 1, point 3).

As we said, the singular fibers of a quadric fibration $(X, L)$ are quadric cones with an isolated singular point. Notice that their number is $[5$, p. 83]:

$$
\mu=2 e-(n+1) b .
$$

We will say that $(X, L)$ is a quadric bundle (conic bundle if $n=2$ ) to mean that $\mu=0$.

\section{(0.2) Hilbert curves}

For the notion and the general properties of the Hilbert curve associated to a polarized manifold we refer to [3]. Here we just recall some basic facts. Let $(X, L)$ be a polarized manifold of dimension $n$ with $K_{X} \not \equiv 0$. For any line bundle $D$ on $X$ consider the expression of the Euler-Poincaré characteristic $\chi(D)$ provided by the Riemann-Roch theorem, and let $p$ be the complexified polynomial of $\chi(D)$, when we set $D=x K_{X}+y L$, with $x, y$ complex numbers, namely $p(x, y)=\chi\left(x K_{X}+y L\right)$. The Hilbert curve of $(X, L)$ is the complex affine plane curve $\Gamma=\Gamma_{(X, L)}$ of degree $n$ defined by $p(x, y)=0$. If the numerical equivalence classes of $K_{X}$ and $L$ are linearly independent, then $\Gamma$ can be regarded as the section of the Hilbert variety of $X$ in the complex affine space $\mathrm{N}(X):=\operatorname{Num}(X) \otimes_{\mathbb{Z}} \mathbb{C}$ with the plane $\left\langle K_{X}, L\right\rangle$ that they span [3, Section 2]. Of course this interpretation fails if $\operatorname{rk}\left\langle K_{X}, L\right\rangle=1$ in $\operatorname{Num}(X)$ : we usually refer to this situation as the degenerate case.

Taking into account that $C:=\frac{1}{2} K_{X}$ is the fixed point of the Serre involution $D \mapsto K_{X}-D$ acting on $\mathrm{N}(X)$, sometimes it is convenient to represent $\Gamma$ in terms of affine coordinates $\left(u=x-\frac{1}{2}, v=y\right)$ centered at $C$ instead of $(x, y)$. In other words, we set $D=\frac{1}{2} K_{X}+E$, where $E=u K_{X}+v L$. Then $\Gamma$ can be represented with respect to these coordinates by $p\left(\frac{1}{2}+u, v\right)=0$. An obvious advantage is that, due to Serre duality, $\Gamma$ is symmetric with respect to $C$ (the origin in the $(u, v)$-plane). We refer to $p\left(\frac{1}{2}+u, v\right)=0$ as the canonical equation of $\Gamma$. Other consequences of the Serre duality are the following facts [3, Section 2]. 1) If $n$ is odd, then $C \in \Gamma ; 2)$ if $n$ is even and $\Gamma \ni C$, then $C$ is a singular point of $\Gamma$. Sometimes, to deal with points at infinity, it is convenient to consider also the projective Hilbert curve $\bar{\Gamma} \subset \mathbb{P}^{2}$, namely the projective closure of $\Gamma$. In this case we use $(x, y, z)$ as homogeneous coordinates on $\mathbb{P}^{2}, z=0$ representing the line at infinity. Given a point $(u, v) \in \mathbb{A}^{2}$, we write $(u: v: 1)$ to denote the same point when regarded as a point of $\mathbb{P}^{2}$. Moreover, we denote by $p_{0}(x, y, z)$ the homogeneous polynomial associated with $p(x, y)$ (i.e. $p(x, y)=p_{0}(x, y, 1)$ ), which defines $\bar{\Gamma}$.

As an example let us describe here the Hilbert curve $\Gamma$ of the quadric fibration in case (i) of Proposition 2. Since $n=2$, according to [3, 3.5], the canonical equation 
of $\Gamma$ can be rewritten, by using coordinates $(u, v)$, as

$$
p\left(\frac{1}{2}+u, v\right)=2(2 u-v)(u-v)
$$

hence, $\Gamma$ is simply the conic reducible in the two lines passing through $C$ whose slopes are 1 and 2 respectively.

\section{The Hilbert Curve of A QUAdric Fibration}

(1.1) Let $(X, L)$ be a quadric fibration over a smooth curve $B$ of genus $q$ via $\pi: X \rightarrow B$. Let $c, e, b$ as in (0.1), let $F \subset X$ a fiber of $\pi$, and denote by $\widetilde{F} \subset P$ the corresponding fiber of $\tilde{\pi}$, so that $F=\widetilde{F} \cdot X$. The following numerical equivalence relations hold: $X \equiv 2 \xi-b \widetilde{F}, K_{X}+(n-1) L \equiv(c+e-b) F$. Moreover, $L=\xi \cdot X$, and the Chern-Wu relation gives $\xi^{n+1}=e$. These relations, evaluating intersections inside $P$, allow us to compute all pluridegrees $d_{i}=K_{X}^{i} \cdot L^{n-i}$ of $(X, L)$, for $i=$ $0, \ldots, n$ (here $\left.d_{0}=d=L^{n}\right)$. First of all,

$$
d=L^{n}=\xi^{n} \cdot(2 \xi-b \widetilde{F})=2 \xi^{n+1}-b \xi^{n} \cdot \widetilde{F}=2 e-b .
$$

Next, for every $i=1, \ldots, n$ we get

$$
\begin{aligned}
d_{i} & =K_{X}^{i} \cdot L^{n-i}=((1-n) \xi+(c+e-b) \widetilde{F})^{i} \cdot \xi^{n-i} \cdot(2 \xi-b \widetilde{F}) \\
& =\left((1-n)^{i} \xi^{i}+i(1-n)^{i-1}(c+e-b) \xi^{i-1} \cdot \widetilde{F}\right) \cdot \xi^{n-i} \cdot(2 \xi-b \widetilde{F}) \\
& =\left((1-n)^{i} \xi^{n}+(1-n)^{i-1} i(c+e-b) \xi^{n-1} \cdot \widetilde{F}\right) \cdot(2 \xi-b \widetilde{F}) \\
& =(1-n)^{i} 2 \xi^{n+1}+\left((1-n)^{i-1} 2 i(c+e-b)-b(1-n)^{i}\right) \xi^{n} \cdot \widetilde{F} \\
& =(1-n)^{i} 2 e+(1-n)^{i-1}(2 i(c+e-b)+b(n-1)) .
\end{aligned}
$$

Thus

$$
d_{i}=(1-n)^{i} 2 e+(1-n)^{i-1}(2 i c+2 i e+(n-1-2 i) b) .
$$

In particular,

$$
\begin{gathered}
d_{1}=K_{X} L^{n-1}=2 c-2 e(n-2)+(n-3) b \\
d_{2}=K_{X}^{2} L^{n-2}=(1-n)(4 c-2(n-3) e-(n-5) b),
\end{gathered}
$$

and

$$
d_{n}=K_{X}^{n}=(1-n)^{n-1}(2 n c+2 e-(n+1) b) .
$$

Finally, note that

$$
\chi:=\chi\left(\mathcal{O}_{X}\right)=1-q
$$

(1.2) Let $\Gamma$ be the Hilbert curve of our quadric fibration $(X, L)$. As $K_{X}+(n-1) L=$ $\pi^{*} \mathcal{A}, \mathcal{A}$ being a line bundle on $B$, we know by $[3$, Theorem 6.1$]$ that $\Gamma$ is defined by

$$
p(x, y)=R(x, y) \prod_{i=1}^{n-2}((n-1) x-y-i)
$$


A. LANTERI

the factor $R$ being a polynomial of degree 2. Call $G$ the conic represented by $R$ and $\ell_{i}(i=1, \ldots, n-2)$ the line corresponding to the $i$ th factor of the most right product in (9). Then $\Gamma=G+\ell_{1}+\cdots+\ell_{n-2}$ consists of the conic $G$ and $n-2$ lines, namely, $\ell_{1}, \ldots, \ell_{n-2}$, which are parallel each other with slope $n-1$ and evenly spaced. Due to the symmetry of $\Gamma$ with respect to the point $C$, the conic has center at $C$, hence,

$$
R(x, y)=\alpha\left(x-\frac{1}{2}\right)^{2}+\beta\left(x-\frac{1}{2}\right) y+\gamma y^{2}+\varepsilon,
$$

for some $\alpha, \beta, \gamma, \varepsilon \in \mathbb{C}$ (in fact $\mathbb{Q}$ ). In terms of coordinates $(u, v)$, simply letting $j=2 i-(n-1)$, we have

$$
p\left(\frac{1}{2}+u, v\right)=\left(\alpha u^{2}+\beta u v+\gamma v^{2}+\varepsilon\right) \prod_{j \equiv n-1(2) ;|j| \leq n-3}\left((n-1) u-v-\frac{j}{2}\right) .
$$

Our aim, is to determine the coefficients $\alpha, \beta, \gamma, \varepsilon$.

Remark. (i) Clearly, if $n=2$, then $\Gamma=G$.

(ii) Note that if $n$ is odd, then one of the lines $\ell_{1}, \ldots, \ell_{n-2}$ (precisely $\ell_{\frac{n-1}{2}}$, the line corresponding to the linear factor with $j=0$ in the last display) contains $C$. On the other hand, if $n$ is even, then no one of the $\ell_{i}$ 's contains $C$; so, if $n$ is even and $C \in \Gamma$, then $C$ is necessarily a singular point of $G$.

Proposition 3. Let $(X, L)$ be an n-dimensional quadric fibration over a smooth curve $B$ of genus $q$, and let $c, e, b, \mu$ be as in (0.1). Then the canonical equation of the Hilbert curve $\Gamma$ of $(X, L)$ in terms of coordinates $(u, v)$ is the following:

$$
\begin{aligned}
p\left(\frac{1}{2}+u, v\right)= & \frac{(-1)^{n}}{n !}\left[(1-n)(2 n c+2 e-(n+1) b) u^{2}\right. \\
& +2(n c-(n-2) e-b) u v+(2 e-b) v^{2} \\
& \left.+\frac{n-1}{4} \mu\right] \prod_{j \equiv n-1(2) ;|j| \leq n-3}\left((n-1) u-v-\frac{j}{2}\right)=0 .
\end{aligned}
$$

Proof. First we determine $\alpha, \beta, \gamma$. To do that we look at the points at infinity of $\Gamma$. Recall that for any divisor $D$ on $X$, the expression provided by the Riemann-Roch theorem has the form $\chi(D)=\frac{1}{n !} D^{n}+\ldots$, where ... stand for terms involving intersections with powers of $D$ lower than $n$. Set $D=x K_{X}+y L$ and let $p_{0}(x, y, z)$ be the homogeneous polynomial associated with $p$, defining $\bar{\Gamma}$ in $\mathbb{P}^{2}$. Restricting to the line at infinity $(z=0)$ and letting $y=1$, we can write

$$
\begin{aligned}
p_{0}(x, 1,0)= & \frac{1}{n !}\left(x K_{X}+L\right)^{n} \\
= & \frac{1}{n !}\left[d_{n} x^{n}+\left(\begin{array}{c}
n \\
1
\end{array}\right) d_{n-1} x^{n-1}+\left(\begin{array}{c}
n \\
2
\end{array}\right) d_{n-2} x^{n-2}+\ldots\right. \\
& \left.\quad \cdots+\left(\begin{array}{c}
n \\
n-2
\end{array}\right) d_{2} x^{2}+\left(\begin{array}{c}
n \\
n-1
\end{array}\right) d_{1} x+d\right] .
\end{aligned}
$$

On the other hand, by (9) and (10) we have

$$
p(x, y)=\left(\alpha\left(x-\frac{1}{2}\right)^{2}+\beta\left(x-\frac{1}{2}\right) y+\gamma y^{2}+\varepsilon\right) \prod_{i=1}^{n-2}((n-1) x-y-i) .
$$


Thus,

$$
\begin{aligned}
p_{0}(x, 1,0)= & \left(\alpha x^{2}+\beta x+\gamma\right)((n-1) x-1)^{n-2} \\
= & \left(\alpha x^{2}+\beta x+\gamma\right)\left[(n-1)^{n-2} x^{n-2}-(n-2)(n-1)^{n-3} x^{n-3}\right. \\
& +\left(\begin{array}{c}
n-2 \\
2
\end{array}\right)(n-1)^{n-4} x^{n-4}+\cdots+(-1)^{n-4}\left(\begin{array}{c}
n-2 \\
2
\end{array}\right)(n-1)^{2} x^{2} \\
& \left.+(-1)^{n-3}(n-2)(n-1) x+(-1)^{n-2}\right] \\
= & \alpha(n-1)^{n-2} x^{n}+(n-1)^{n-3}((n-1) \beta-(n-2) \alpha) x^{n-1} \\
& +(n-1)^{n-4}\left((n-1)^{2} \gamma-(n-1)(n-2) \beta+\left(\begin{array}{c}
n-2 \\
2
\end{array}\right) \alpha\right) x^{n-2} \\
& +\cdots+(-1)^{n-3}((n-2)(n-1) \gamma-\beta) x+(-1)^{n-2} \gamma .
\end{aligned}
$$

For every power of $x$ we can thus equate the coefficients in the above expression with those in (12). In particular, looking at the terms of degrees $n, 1$ and 0 , we get the following equalities

$$
\begin{gathered}
\frac{1}{n !} d_{n}=(n-1)^{n-2} \alpha, \\
\frac{1}{n !} n d_{1}=(-1)^{n-3}(n-2)(n-1) \gamma+(-1)^{n-2} \beta, \\
\frac{1}{n !} d=(-1)^{n-2} \gamma
\end{gathered}
$$

Taking into account (3), (5), (7), they give

$$
\begin{gathered}
\alpha=\frac{(-1)^{n}}{n !}(1-n)[2 n c+2 e-(n+1) b], \\
\beta=\frac{(-1)^{n}}{n !}[2 n c-2(n-2) e-2 b], \\
\gamma=\frac{(-1)^{n}}{n !}[2 e-b] .
\end{gathered}
$$

Next we determine $\varepsilon$. By Serre duality, recalling (8), we get

$$
p(1,0)=\chi\left(K_{X}\right)=(-1)^{n} \chi\left(\mathcal{O}_{X}\right)=(-1)^{n}(1-q)=(-1)^{n-1} \frac{c}{2} .
$$

On the other hand, (9) and (10) show that

$$
\begin{aligned}
p(1,0) & =\left(\frac{1}{4} \alpha+\varepsilon\right) \prod_{1=1}^{n-2}(n-1-i) \\
& =\left(\frac{1}{4} \alpha+\varepsilon\right)(n-2) !
\end{aligned}
$$

By comparing these two values we obtain

$$
\varepsilon=-\frac{1}{4} \alpha+\frac{(-1)^{n-1}}{(n-2) !} \frac{c}{2},
$$


and recalling (13) and (2) this gives

$$
\begin{aligned}
\varepsilon & =-\frac{1}{4} \frac{(-1)^{n}}{n !}(1-n)(2 n c+2 e-(n+1) b)-\frac{(-1)^{n}}{(n-2) !} \frac{c}{2} \\
& =(-1)^{n}\left[\frac{n-1}{4 n !}(2 n c+2 e-(n+1) b)-\frac{1}{(n-2) !} \frac{c}{2}\right] \\
& =(-1)^{n}\left[\frac{c}{2}\left(\frac{n(n-1)}{n !}-\frac{1}{(n-2) !}\right)+\frac{1}{n !}\left(\frac{n-1}{2} e-\frac{1}{4}\left(n^{2}-1\right) b\right)\right] \\
& =\frac{(-1)^{n}}{n !}(n-1)\left[\frac{1}{2} e-\frac{1}{4}(n+1) b\right] \\
& =\frac{(-1)^{n}}{n !}(n-1) \frac{\mu}{4} .
\end{aligned}
$$

This concludes the proof.

We emphasize that the equation of $\Gamma$ in Proposition 3 holds for any quadric fibration $(X, L)$ : actually, we did not require that $\operatorname{rk}\left\langle K_{X}, L\right\rangle=2$. As we said, $\Gamma$ consists of $n-2$ evenly spaced parallel lines $\ell_{i}(i=1, \ldots, n-2)$ of equation $(n-1) u-v+\frac{n-1-2 i}{2}=0$ plus a conic $G$, we are going to describe now. To do that, let us come back to our cases i), ii), iii) in (0.1).

As a consequence of Proposition 3 , just dividing the first factor in the expression of $p\left(\frac{1}{2}+u, v\right)$ by $\frac{(-1)^{n}}{n !}$, we get the following

Corollary 4. Let $(X, L)$ be any quadric fibration over a smooth curve $B$, and let $G$ be the complement of the lines $\ell_{1}, \ldots, \ell_{n-2}$ in $\Gamma_{(X, L)}$. Then the quadratic form defining $G$ in coordinates $(u, v)$, is given by the following symmetric matrix:

$$
A=\left(\begin{array}{cc}
A_{\infty} & 0 \\
0 & (n-1) \frac{\mu}{4}
\end{array}\right),
$$

where

$$
A_{\infty}=\left(\begin{array}{cc}
(1-n)(2 n c+2 e-(n+1) b) & n c-(n-2) e-b \\
n c-(n-2) e-b & 2 e-b
\end{array}\right) .
$$

Case i) in (0.1) was already discussed at the end of Section 0, so, before considering the general case let us discuss here case ii). In this case $a=c+e-b=0$, hence $b=e+c$. By using this equality, a straightforward verification shows that $A_{\infty}$ takes the form

$$
A_{\infty}=(e-c)\left(\begin{array}{cc}
(n-1)^{2} & -(n-1) \\
-(n-1) & 1
\end{array}\right) .
$$

First of all we point out the following fact.

Lemma 5. $e-c>0$.

Proof. Recall that in case ii) $(X, L)$ is either a del Pezzo surface with $L=-K_{X}$ and $X \neq \mathbb{P}^{2}$, or $\left(\mathbb{P}^{1} \times \mathbb{P}^{1} \times \mathbb{P}^{1}, \mathcal{O}(1,1,1)\right)$; so $B \cong \mathbb{P}^{1}$ in both cases, and then $c=-2$. Thus, $e-c=e+2$. If $L$ is very ample, then $e \geq 0$, since $\mathcal{E}=\pi_{*} L$ is spanned (e.g., see [10, beginning of p. 509]), hence $e-c \geq 2$. Suppose now that $L$ is not very ample. In this case we note that $\mathcal{E}$ is not ample, otherwise, $L=\xi_{X}$ would be very ample, so being $\xi$. Thus we cannot claim that $e>0$. However, according to what we said before, this situation occurs only when $(X, L)$ is a del Pezzo surface with 
$L=-K_{X}$ of degree $d=K_{X}^{2} \leq 2$. Since $X$ is isomorphic to $\mathbb{P}^{2}$ blown-up at $9-d$ points in general position, and $\pi: X \rightarrow \mathbb{P}^{1}$ is induced by the pencil of lines through one of the $p_{i}$ 's, we deduce that $X$ has exactly $\mu=8-d$ singular fibers. Then (2) says that $2 e-3 b=8-d$. On the other hand,

$$
d=L^{2}=\left(\xi_{X}\right)^{2}=\xi^{2} \cdot X=\xi^{2} \cdot(2 \xi-b \widetilde{F})=2 \xi^{3}-b \xi^{2} \cdot \widetilde{F}=2 e-b,
$$

since $\xi^{3}=\operatorname{deg} \mathcal{E}=e$ by the Chern-Wu relation. By subtracting the latter equation from the former we thus get $d-2 b=8-d$, hence $b=d-4$ and so $e=\frac{1}{2}(b+d)=$ $d-2 \geq-1$. Therefore, $e-c>0$ even in this case.

Now, in view of the above expression of the matrix $A, G$ has the following equation:

$$
(e-c)((n-1) u-v)^{2}+(n-1) \frac{\mu}{4}=0 .
$$

Thus, the above Lemma and the fact that $\mu \geq 0$ imply that $G$ is imaginary (the polynomial on the left hand is the product of two complex conjugate linear factors and $\left.G_{\mathbb{R}}=\emptyset\right)$, except when $\mu=0$. Moreover, recalling what we proved in (0.1), this situation occurs if and only if either

1) $n=2$ and $(X, L)=\left(\left(\mathbb{P}^{1} \times \mathbb{P}^{1}, \mathcal{O}(2,2)\right)\right.$ or $\left(\mathbb{F}_{1},-K_{\mathbb{F}_{1}}\right)$, or

2) $n=3$ and $(X, L)=\left(\mathbb{P}^{1} \times \mathbb{P}^{1} \times \mathbb{P}^{1}, \mathcal{O}(1,1,1)\right)$.

For both pairs in case 1$), \Gamma_{(X, L)}=G$ is described by $4(u-v)^{2}=0$, while in case 2$)$, $\Gamma_{(X, L)}$ has canonical equation $-2(u-v)^{3}=0[3,2.5]$. This concludes the analysis of case ii).

Now let us consider the general case, namely case iii). First of all, from (17) we have

$$
\operatorname{det} A_{\infty}=-n^{2}(c+e-b)^{2} .
$$

Recalling (1) and the related discussion in (0.1), we conclude that $c+e-b=$ $\operatorname{deg}\left(K_{B}+\operatorname{det} \mathcal{E}-\mathcal{B}\right)>0$, since $\operatorname{rk}\left\langle K_{X}, L\right\rangle=2$. Therefore,

$$
\operatorname{det} A_{\infty} \neq 0 \text {, }
$$

in case iii), so that $G$ is of hyperbolic type (also from the real point of view, since in fact $\left.\operatorname{det} A_{\infty}<0\right)$. Moreover,

$$
\operatorname{det} A=(n-1) \frac{\mu}{4} \operatorname{det} A_{\infty}
$$

hence, $G$ is reducible if and only if $\mu=0$, i.e., $(X, L)$ has no singular fibers. By $(2)$, this is equivalent to $2 e=(n+1) b$, hence, when reducible, $G$ has equation

$$
\begin{aligned}
{\left[\begin{array}{ll}
u & v
\end{array}\right] A_{\infty}\left[\begin{array}{c}
u \\
v
\end{array}\right] } & =2 n\left[(1-n) c u^{2}+\left(c-\frac{n-1}{n+1} e\right) u v-\frac{e}{n+1} v^{2}\right] \\
& =2 n\left[c u((1-n) u+v)+\frac{e}{n+1} v((1-n) u+v)\right] \\
& =2 n((1-n) u+v)\left(c u+\frac{e}{n+1} v\right)=0 .
\end{aligned}
$$

As a consequence, the directions of the two lines meeting at $C$, constituting $G$, are represented by the points at infinity

$$
P_{\infty}=(1: n-1: 0) \text { and } Q_{\infty}=(-e:(n+1) c: 0) .
$$

Clearly, they are distinct, due to (18). Note that the first one represents also the direction of the lines $\ell_{i}, i=1, \ldots, n-2$. So, if $n$ is odd then $\Gamma$ is non-reduced, since 
the line $\ell_{\underline{n-1}}$ appears as a component of multiplicity 2 (see Remark (ii)). Moreover, $C$ is a triple point for $\Gamma$.

This provides a complete generalization of [3, Proposition 4.8].

\section{Characterizing $\mathbb{Q}$-Fibrations via Hilbert curves}

Here is a characterization of $\mathbb{Q}$-fibrations over a curve in terms of their Hilbert curves. It can be regarded as an analogue of the characterization of scrolls provided in $[9$, Section 4]. As there, we need to assume that $(X, L)$ is not as in the degenerate case.

Theorem 6. Let $(X, L)$ a polarized manifold of dimension $n \geq 3$ with $\operatorname{rk}\left\langle K_{X}, L\right\rangle=$ 2 and suppose that it is not a scroll over a smooth curve. If $\Gamma_{(X, L)}$ has an equation as in (11), then $(X, L)$ is a quadric fibration (in the adjunction theoretic sense) over a smooth curve.

Proof. By using both assumptions together, we see that $K_{X}+(n-1) L$ is nef [2, Theorem 7.2.5]. Hence, by the Kawamata-Shokurov base point free theorem, $\left|m\left(K_{X}+(n-1) L\right)\right|$ defines a morphism $\Phi: X \rightarrow \mathbb{P}^{N}$ for some integer $m>>0$. Use the same notation as in the proof of Proposition 3: if $\Gamma$ is described by (11), then its projective closure contains the point at infinity $P_{\infty}=(1: n-1: 0)$ (with multiplicity $\geq n-2)$; therefore, $p_{0}(1, n-1,0)=p_{0}\left(\frac{1}{n-1}, 1,0\right)=0$. Recalling the expression of $\chi$, we thus get

$$
0=p_{0}\left(\frac{1}{n-1}, 1,0\right)=\frac{1}{n !}\left(\frac{1}{n-1} K_{X}+L\right)^{n}
$$

hence $\left(K_{X}+(n-1) L\right)^{n}=0$. It follows that $K_{X}+(n-1) L$ is nef but not big and then, according to [2, Theorem 7.3.2], $(X, L)$ must be one of the following:

1) a del Pezzo manifold,

2) a quadric fibration over a smooth curve (in the adjunction theoretic sense), or

3) a scroll over a surface.

We need to show that we are in case 2). Clearly, case 1) cannot occur, since $\operatorname{rk}\left\langle K_{X}, L\right\rangle=2$. To rule out case 3 ) it is enough to show that the morphism defined by $\Phi$ via Stein factorization, has a 1-dimensional image. To do that, in view of the ampleness of $L$, we will show that

$$
\left(K_{X}+(n-1) L\right)^{2} \cdot L^{n-2}=0
$$

Note that

$$
\left(K_{X}+(n-1) L\right)^{2} \cdot L^{n-2}=d_{2}+2(n-1) d_{1}+(n-1)^{2} d
$$

On the other hand, by equating the coefficients of terms of degrees 2, 1 and 0 in $x$ in (12) with the corresponding ones in the expression of $p_{0}(x, 1,0)$ after $(12)$, we can express $d_{2}, d_{1}$ and $d$ in terms of the coefficients $\alpha, \beta$ and $\gamma$ appearing in (10). The two equations before (13) provide such expressions for $d_{1}$ and $d$. Similarly, we obtain

$$
\frac{1}{n !}\left(\begin{array}{l}
n \\
2
\end{array}\right) d_{2}=(-1)^{n}\left(\alpha-(n-1)(n-2) \beta+2\left(\begin{array}{c}
n-2 \\
2
\end{array}\right)(n-1)^{2} \gamma\right)
$$


Replacing these expressions in (19) a straightforward verification shows that

$$
\left(K_{X}+(n-1) L\right)^{2} \cdot L^{n-2}=2(-1)^{n}(n-2) !\left(\alpha+(n-1) \beta+(n-1)^{2} \gamma\right) .
$$

Finally, recalling the expressions of $\alpha, \beta, \gamma$ provided by (13), (14), (15), we can directly check that

$$
\alpha+(n-1) \beta+(n-1)^{2} \gamma=0
$$

as required.

Remark. The characters $q, e, b, \mu$ are not uniquely determined by the coefficients of the polynomial $R$ in (10). Clearly, $\mu$ is determined by the constant term $\varepsilon$ of $R$ in view of (16), but the fact that $\mu \geq 0$ puts the restriction that $\varepsilon \geq 0$ or $\leq 0$ according to whether $n$ is even or odd respectively. On the other hand, to recover $c, e, b$ we have to solve the linear system

$$
\left\{\begin{array}{cl}
2 n c+2 e-(n+1) b & =(-1)^{n} n ! \frac{\alpha}{1-n} \\
n c-(n-2) e-b & =(-1)^{n} n ! \frac{\beta}{2} \\
2 e-b & =(-1)^{n} n ! \gamma .
\end{array}\right.
$$

Denoting by $E_{i}, i=1,2,3$ the above equations respectively, we immediately see that $E_{2}, E_{3}$ are linearly independent, while

$$
E_{1}=2 E_{2}+(n-1) E_{3}
$$

hence the solutions are those of $E_{2}$ and $E_{3}$, namely,

$$
(c, e, b)=\left(c, c+(-1)^{n-1}(n-1) !\left(\frac{\beta}{2}-\gamma\right), 2 c+(-1)^{n-1}(n-1) !(\beta+(n-2) \gamma)\right)
$$

\section{More ON THE CONIC $G$}

In connection with [3, Problem $6.6(1)]$ it is natural to ask the following question about the conic $G$ arisen at the end of Section 1. Can $G$ be the Hilbert curve of some polarized surface $(S, \mathcal{L})$, somehow related to $(X, L)$, hence to $B$ ? This question is meaningful for $n \geq 3$, and the answer is non-obvious at all: this case will be discussed in this Section, while for the natural modification in case $n=2$, where $G=\Gamma_{(X, L)}$, we refer to Section 4. According to the experience deriving from [9, Remark 4.1] one could ask, more generally, if $G$ can be the Hilbert curve of a $\mathbb{Q}$-polarized surface. In fact the first step of the discussion below is done looking at this more general framework; however, an argument relying on genus formula and adjunction, we need at a certain point, will compel us to confine to the case in which $(S, \mathcal{L})$ is a polarized surface. Moreover, in order to produce an effective result, we will assume that $(X, L)$ is a $\mathbb{Q}$-bundle, i.e., $\mu=0$. In spite of this more restricted setting, the output is enough to realize that, in general, the above question has a negative answer.

So, let $(X, L)$ be a quadric bundle of dimension $n \geq 2$ over a smooth curve $B$ of genus $q$. Recall that $c=2 q-2$; moreover, $2 e=(n+1) b$ by $(2)$, hence the matrix $A=\left[a_{i j}\right]$ of $G$ specializes to the following matrix:

$$
A=n\left(\begin{array}{ccc}
4(1-n)(q-1) & 2(q-1)-\frac{n-1}{2} b & 0 \\
2(q-1)-\frac{n-1}{2} b & b & 0 \\
0 & 0 & 0
\end{array}\right)
$$


Now let $(S, \mathcal{L})$ be any $\mathbb{Q}$-polarized surface and let $D=x K_{S}+y \mathcal{L}$. Rewriting $D$ as $\left(\frac{1}{2}+u\right) K_{X}+v \mathcal{L}$ we see that the Hilbert curve of $(S, \mathcal{L})$ is the conic, defined in terms of coordinates $(u, v)$, by the following equation

$$
\begin{aligned}
p\left(\frac{1}{2}+u, v\right) & =\chi\left(\mathcal{O}_{S}\right)+\frac{1}{2}\left(\left(u+\frac{1}{2}\right) K_{S}+v \mathcal{L}\right)\left(\left(u-\frac{1}{2}\right) K_{S}+v \mathcal{L}\right) \\
& =\frac{1}{2}\left(K_{S}^{2} u^{2}+2 K_{S} \cdot \mathcal{L} u v+\mathcal{L}^{2} v^{2}+2 \chi\left(\mathcal{O}_{S}\right)-\frac{1}{4} K_{S}^{2}\right)=0 .
\end{aligned}
$$

Hence, up to a multiplicative constant, its matrix $A^{\prime}=\left[a_{i j}^{\prime}\right]$ is the following

$$
A^{\prime}=\left(\begin{array}{ccc}
K_{S}^{2} & K_{S} \cdot \mathcal{L} & 0 \\
K_{S} \cdot \mathcal{L} & \mathcal{L}^{2} & 0 \\
0 & 0 & 2 \chi\left(\mathcal{O}_{S}\right)-\frac{1}{4} K_{S}^{2}
\end{array}\right)
$$

So, if $G$ is the Hilbert curve of $(S, \mathcal{L})$ there must exist a nonzero constant $\rho \in \mathbb{Q}$ such that

$$
A=\rho A^{\prime}
$$

In particular, note that

$$
a_{22}=n b=2 e-b=L^{n}>0 .
$$

Since $a_{22}^{\prime}=\mathcal{L}^{2}>0$ the equality $a_{22}=\rho a_{22}^{\prime}$ says that $\rho>0$. Moreover, since $0=a_{33}=\rho a_{33}^{\prime}$ we see that

$$
K_{S}^{2}=8 \chi\left(\mathcal{O}_{S}\right)
$$

Furthermore, looking at both matrices we have

$$
4 n(1-n)(q-1)=a_{11}+4 a_{33} \quad \text { and } \quad 8 \chi\left(\mathcal{O}_{S}\right)=a_{11}^{\prime}+4 a_{33}^{\prime} .
$$

Therefore,

$$
4 n(n-1)(1-q)=\rho 8 \chi\left(\mathcal{O}_{S}\right) .
$$

Since $n \geq 2$ the above equality shows the following equivalences:

$$
\begin{array}{lll}
q=1 & \text { if and only if } & \chi\left(\mathcal{O}_{S}\right)=0 ; \\
q=0 & \text { if and only if } & \chi\left(\mathcal{O}_{S}\right)>0 ; \\
q \geq 2 & \text { if and only if } & \chi\left(\mathcal{O}_{S}\right)<0 .
\end{array}
$$

This suggests to split the analysis according to the above tricothomy.

As announced, from now on in this Section we assume that $n \geq 3$ and that $(S, \mathcal{L})$ is a polarized surface. Let $g^{\prime}=g(S, \mathcal{L})$ be its sectional genus. Then $g^{\prime} \geq 0$, and

$$
n\left(2 q-2+\frac{3-n}{2} b\right)=a_{12}+a_{22}=\rho\left(a_{12}^{\prime}+a_{22}^{\prime}\right)=\rho\left(2 g^{\prime}-2\right) .
$$

Now we are ready to discuss the three cases above separately.

Case (a): $q=1$. This case is settled by the following Proposition.

Proposition 7. Let $(X, L)$ be a quadric bundle of dimension $n \geq 3$ over a smooth curve of genus 1 . Then $G$ is the Hilbert curve of a polarized surface $(S, \mathcal{L})$ if and only if $n=3$ and $(S, \mathcal{L})$ is an elliptic scroll. 
Proof. Let $G=\Gamma_{(S, \mathcal{L})}$. We have $\chi\left(\mathcal{O}_{S}\right)=0$, since $q=1$. Moreover, $K_{S}^{2}=0$, by (23), and $b>0$ by (22). We thus see from

$$
\rho K_{S} \cdot \mathcal{L}=a_{12}=-\frac{n(n-1) b}{2}
$$

that $K_{S} \cdot \mathcal{L}<0$, since $\rho>0$. Due the ampleness of $\mathcal{L}$ this implies that no positive multiple of $K_{S}$ can be effective, hence $S$ is a ruled surface in view of the Enriques theorem [1, Theorem VI.17]. Combining this with $\chi\left(\mathcal{O}_{S}\right)=0$ and $K_{S}^{2}=0$, we conclude that $S$ is a $\mathbb{P}^{1}$-bundle over a smooth curve of genus 1 . Of course $\mathcal{L}$ induces $\mathcal{O}_{\mathbb{P}^{1}}(r)$ on every fiber of $S$, for some positive integer $r$. Then $G$ is the Hilbert curve of $(S, \mathcal{L})$ if and only if its projective closure contains the point at infinity $(r: 2: 0)$, according to [9, Proposition 3.1]. Note that $\Gamma$ has equation: $n b\left(-(n-1) u v+v^{2}\right)=0$. Hence the above condition is expressed by

$$
(n-1) r=2 .
$$

Due to the assumption $n \geq 3$, this can occur if and only if $(n, r)=(3,1)$. Finally, note that $\mathcal{L}^{2}=-K_{S} \cdot \mathcal{L}$ for any elliptic surface scroll, in view of the genus formula. Hence the Hilbert curve of any elliptic surface scroll is the component $G$ of the Hilbert curve of a 3-dimensional quadric bundle over an elliptic curve.

Case (b): $q=0$. We have

Proposition 8. Let $(X, L)$ be a rational quadric bundle of dimension $n \geq 3$. Then $G$ is the Hilbert curve of a polarized surface $(S, \mathcal{L})$ if and only if $n=3$ and $(S, \mathcal{L})$ is a rational scroll (including $\left(\mathbb{P}^{1} \times \mathbb{P}^{1}, \mathcal{O}(1,1)\right)$ ).

Proof. Let $G=\Gamma_{(S, \mathcal{L})}$. We know that $\chi\left(\mathcal{O}_{S}\right)>0$, hence $K_{S}^{2}>0$ by (23). From (24) we get

$$
\rho=\frac{n(n-1)}{2 \chi\left(\mathcal{O}_{S}\right)} .
$$

On the other hand $b>0$ by (22). Taking into account (26), formula (25) shows that

$$
g^{\prime}-1=-\frac{\chi\left(\mathcal{O}_{S}\right)}{(n-1)}\left(2+\frac{(n-3) b}{2}\right) .
$$

Since $n \geq 3$ and $b>0$, the right hand side of $(27)$ is negative, hence $g^{\prime}=0$. Then the classification of polarized surfaces with sectional genus zero and (23) say that $(S, \mathcal{L})$ is a rational scroll (including $\left(\mathbb{P}^{1} \times \mathbb{P}^{1}, \mathcal{O}(1,1)\right)$ ); in particular, $\chi\left(\mathcal{O}_{S}\right)=1$. Replacing this value in (27) we thus get the equality

$$
(n-3)\left(1-\frac{b}{2}\right)=0 .
$$

So, either $n=3$, as claimed in the statement, or $b=2$ and $n \geq 4$. However, in the latter case (22) would give $a_{22}=2 n$, hence, recalling (26) the degree of $(S, \mathcal{L})$ would be

$$
\mathcal{L}^{2}=a_{22}^{\prime}=\frac{1}{\rho} 2 n=\frac{4}{n-1} .
$$

Since $n \geq 4$, this can occur only for $n=5$, giving $\mathcal{L}^{2}=1$, but this is impossible, since there are no rational surface scrolls of degree 1 . Conversely, for any $(S, \mathcal{L})$ as in the statement we have $g^{\prime}=0$, hence $n=3$ by $(27)$. 
Case (c): $q \geq 2$. In this case we have the following result.

Proposition 9. Let $(X, L)$ be a quadric bundle of dimension $n \geq 3$ over a smooth curve of genus $q \geq 2$. Then $G$ is the Hilbert curve of a polarized surface $(S, \mathcal{L})$ if and only if $n=3$ and $(S, \mathcal{L})$ is a scroll over a smooth curve of genus $q^{\prime} \geq 2$ with $\left(q^{\prime}-1,3 \mathcal{L}^{2}\right)$ proportional to $\left(q-1, L^{3}\right)$.

Proof. Suppose that $G$ is the Hilbert curve of some polarized surface $(S, \mathcal{L})$. Here $\chi\left(\mathcal{O}_{S}\right)<0$, hence $S$ is ruled by the Castelnuovo-de Franchis theorem [1, Theorem X.4]. Thus $\chi\left(\mathcal{O}_{S}\right)=1-q^{\prime}<0$, where $q^{\prime} \geq 2$ denotes the irregularity. Moreover, (23) says that $S$ is a $\mathbb{P}^{1}$-bundle over a smooth curve of genus $q^{\prime}$. Now, (24) gives

$$
\rho=-\frac{n(n-1)(q-1)}{2 \chi\left(\mathcal{O}_{S}\right)} .
$$

By (25), recalling (28), we thus get

$$
g^{\prime}-1=\left(2(q-1)-\frac{(n-3) b}{2}\right) \frac{q^{\prime}-1}{(n-1)(q-1)} .
$$

Since $q^{\prime} \geq 2$ we note that $(S, \mathcal{L})$ is a scroll if and only if $g^{\prime}=q^{\prime}$. Thus $(29)$ shows that $(S, \mathcal{L})$ is a scroll if $n=3$. Conversely, if $(S, \mathcal{L})$ is a scroll, letting $g^{\prime}=q^{\prime},(29)$ gives $(n-1)(q-1)=2(q-1)+\frac{(3-n)}{2} b$, i.e.,

$$
(n-3)\left(q-1+\frac{b}{2}\right)=0 \text {. }
$$

Then $n=3$, since the second factor in the above equality is positive, because $q \geq 2$ and $b>0$ in view of (22). We can thus proceed assuming that $n \geq 4$ and that $(S, \mathcal{L})$ is not a scroll. It thus follows that $K_{S}+\mathcal{L}$ is nef, hence $\left(K_{S}+\mathcal{L}\right)^{2} \geq 0$. By combining this with the genus formula and the fact that $S$ is a $\mathbb{P}^{1}$-bundle we get

$$
0 \leq K_{S}^{2}+2 K_{S} \cdot \mathcal{L}+\mathcal{L}^{2}=8\left(1-q^{\prime}\right)+4\left(g^{\prime}-1\right)-\mathcal{L}^{2}<4\left(g^{\prime}-2 q^{\prime}+1\right) .
$$

Hence $g^{\prime} \geq 2 q^{\prime}$. On the other hand, since $b>0$ and $n \geq 4$, (29) shows that

$$
g^{\prime}-1<\frac{2\left(q^{\prime}-1\right)}{n-1}
$$

But, comparing this with the above inequality we immediately get a contradiction. To complete the proof it remains to relate $q$ to the genus $q^{\prime}$ of the base curve of $(S, \mathcal{L})$. This follows from $(28)$ and the relation $L^{n}=n b=a_{2} 2=\rho a_{22}^{\prime}=\rho \mathcal{L}^{2}$, letting $n=3$. We obtain $q^{\prime}-1=\frac{3 \mathcal{L}^{2}}{L^{3}}(q-1)$.

\section{The CASE OF CONIC Fibrations}

In this Section we specialize the discussion to case $n=2$, proving some miscellaneous results. So, let $(X, L)$ be a conic fibration over a smooth curve $B$ of genus $q$. Its degree $d$ and the number $\mu$ of its singular fibers can be expressed in terms of the numerical characters introduced in Section 1 , by $d=L^{2}=2 e-b$ and $\mu=2 e-3 b$. Thus, the Hilbert curve $\Gamma$ of $(X, L)$ is the conic represented by the matrix

$$
A=\left(\begin{array}{ccc}
8(1-q)-\mu & 4(q-1)-b & 0 \\
4(q-1)-b & 2 e-b & 0 \\
0 & 0 & \frac{\mu}{4}
\end{array}\right) .
$$

Every singular fiber of $X$ consists of two components, each being a (-1)-curve. Choose one of them, say $e_{i}$, for $i=1, \ldots, \mu$. By contracting these $\mu(-1)$-curves 
we get a birational morphism $\eta: X \rightarrow X_{0}$, where $X_{0}$ is a $\mathbb{P}^{1}$-bundle over $B$. Let $L_{0}$ be the line bundle on $X_{0}$ such that $L=\eta^{*} L_{0}-\sum_{i=1}^{\mu} e_{i}$. Then $L_{0}$ is ample by the Nakai-Moishezon criterion, hence $\left(X_{0}, L_{0}\right)$ is a polarized surface, which is a conic bundle over $B$. Since $K_{X}=\eta^{*} K_{X_{0}}+\sum_{i=1}^{\mu} e_{i}$ we have

$$
K_{X}^{2}=K_{X_{0}}^{2}-\mu=8(1-q)-\mu, \quad K_{X} \cdot L=K_{X_{0}} \cdot L_{0}+\mu, \quad L^{2}=L_{0}^{2}-\mu,
$$

and therefore the matrix $A$ of $\Gamma$ can be rewritten as

$$
A=\left(\begin{array}{ccc}
K_{X_{0}}^{2}-\mu & K_{X_{0}} \cdot L_{0}+\mu & 0 \\
K_{X_{0}} \cdot L_{0}+\mu & L_{0}^{2}-\mu & 0 \\
0 & 0 & \frac{\mu}{4}
\end{array}\right) .
$$

In other words,

$$
A=A_{0}+\mu U,
$$

where $A_{0}$ is the matrix of the conic which is the Hilbert curve of any conic bundle with the same numerical characters as $\left(X_{0}, L_{0}\right)$, and

$$
U=\left(\begin{array}{ccc}
-1 & 1 & 0 \\
1 & -1 & 0 \\
0 & 0 & \frac{1}{4}
\end{array}\right)
$$

Let $\gamma$ be the conic corresponding to $U$. The above discussion proves the following fact.

Proposition 10. Let $(X, L)$ be a conic fibration over a smooth curve, with $\mu$ singular fibers, and let $\left(X_{0}, L_{0}\right)$ be a conic bundle constructed as before. Then the Hilbert curve of $(X, L)$ is the conic determined by $(1: \mu)$ in the pencil generated by the Hilbert curve of any conic bundle with the same characters as $\left(X_{0}, L_{0}\right)$ and $\gamma$.

As to the natural question whether $\gamma$ is the Hilbert curve of some polarized surface, the answer is negative. In fact we can say more.

Lemma 11. The conic $\gamma$ cannot be the Hilbert curve of any $\mathbb{Q}$-polarized surface.

Proof. By contradiction, suppose that $\gamma=\Gamma_{(S, \mathcal{L})}$ for some $\mathbb{Q}$-polarized surface $(S, \mathcal{L})$. Then there exist a nonzero rational number $\rho$ such that

$$
\left(-1,1,-1, \frac{1}{4}\right)=\rho\left(K_{S}^{2}, K_{S} \cdot L, L^{2}, 2 \chi\left(\mathcal{O}_{S}\right)-\frac{1}{4} K_{S}^{2}\right) .
$$

The third condition implies that $\rho<0$ and then the first one says that $K_{S}^{2}>0$. On the other hand, taking into account the first one again, the fourth condition becomes

$$
\frac{1}{4}=2 \rho \chi\left(\mathcal{O}_{S}\right)-\frac{1}{4} \rho K_{S}^{2}=2 \rho \chi\left(\mathcal{O}_{S}\right)+\frac{1}{4} .
$$

Therefore $\chi\left(\mathcal{O}_{S}\right)=0$, but then the Enriques-Kodaira classification of algebraic surfaces shows that, necessarily, $K_{S}^{2} \leq 0$, a contradiction.

Now we come back to the problem addressed in Section 3 in case $n \geq 3$, dealing with its appropriate modification for $n=2$. So, let $(X, L)$ be a conic bundle over a smooth curve $B$ of genus $q$, and let $\Gamma:=\Gamma_{(X, L)}$ be its Hilbert curve. Suppose that $(S, \mathcal{L})$ is another polarized surface having $\Gamma$ as its Hilbert curve. What about $(S, \mathcal{L})$ ? Since conic bundles are $\mathbb{P}^{1}$-bundles, this case fits into [9, Proposition 3.1]; we note, however, that the present question was not explicitly discussed there. First 
of all, specializing the matrix $A=\left[a_{i j}\right]$ in Section 3 to case $n=2$ we see that $\Gamma$ has the following equation

$$
4(1-q) u^{2}+(4(q-1)-b) u v+b v^{2}=(4(1-q) u-b v)(u-v)=0 .
$$

Hence, its points at infinity are

$$
P_{\infty}=(1: 1: 0) \quad \text { and } \quad Q_{\infty}=(b: 4(1-q): 0) .
$$

Looking at $\Gamma$ from the real point of view, we point out that for $q \geq 1$ only $P_{\infty}$ corresponds to a direction interior to the first quadrant, while this is true for both $P_{\infty}$ and $Q_{\infty}$ if $q=0$.

As already said, another polarized surface $(S, \mathcal{L})$ admits $\Gamma$ as Hilbert curve if and only if there exists a non-zero rational number $\rho$ such that $A=\rho A^{\prime}$, where $A^{\prime}=\left[a_{i j}^{\prime}\right]$ is as in (20). The discussion we made in Section 3 leading to the tricothomy $q \geq 2$, $q=1, q=0$, is still valid; moreover, in each case we can see that $S$ is a $\mathbb{P}^{1}$-bundle over a smooth curve of genus $q^{\prime}$, where $q^{\prime} \geq 2, q^{\prime}=1$, or $q^{\prime}=0$ respectively. Let $r$ be the positive integer such that $\mathcal{L}$ induces $\mathcal{O}_{\mathbb{P}^{1}}(r)$ on every fiber of $S$. Then, according to $[9$, Proposition 3.1] the projective closure of $\Gamma$ must contain the point at infinity $P_{\infty}^{\prime}=(r: 2: 0)$, which corresponds to a direction interior to the first quadrant. So, if $q \geq 2$, it follows that $P_{\infty}^{\prime}=P_{\infty}$ and then $Q_{\infty}$ must coincide with the point $P_{\infty}^{\prime \prime}=\left(\mathcal{L}^{2}, 4 r\left(1-q^{\prime}\right): 0\right)\left[9\right.$, p. 11]. Thus, $P_{\infty}^{\prime}=(r: 2: 0)=(1: 1: 0)=P_{\infty}$ implies $r=2$, while $P_{\infty}^{\prime \prime}=\left(\mathcal{L}^{2}: 8\left(1-q^{\prime}\right): 0\right)=(b: 4(1-q): 0)=Q_{\infty}$, recalling that $L^{2}=2 b$, implies $\frac{L^{2}}{\mathcal{L}^{2}}=\frac{1-q}{1-q^{\prime}}$ only. Therefore, for $q \geq 2$, any polarized surface $(S, \mathcal{L})$ having the same Hilbert curve as $(X, L)$ is in turn a conic bundle, of degree $d^{\prime}$, over a smooth curve of genus $q^{\prime}$ and $\left(d^{\prime}, 1-q^{\prime}\right)$ is proportional to $(d, 1-q)$ (cf. [9, Remark 3.1]).

In the same way, if $q=1$, we see from (30) that $P_{\infty}^{\prime}=P_{\infty}$, i.e., $r=2$. Therefore $(S, \mathcal{L})$ is an elliptic conic bundle. Note however that its degree is arbitrary.

Finally, let $q=0$. From $8=a_{11}=\rho a_{11}^{\prime}=\rho K_{S}^{2}=8 \rho$ we get $\rho=1$. Moreover, both points $P_{\infty}$ and $Q_{\infty}$ in (30) correspond to directions interior to the first quadrant. So we have two possibilities: either a) $P_{\infty}^{\prime}=P_{\infty}$ and $P_{\infty}^{\prime \prime}=Q_{\infty}$, or b) $P_{\infty}^{\prime}=Q_{\infty}$ and $P_{\infty}^{\prime \prime}=P_{\infty}$. In case a) we get $r=2$ and $L^{2}=\mathcal{L}^{2}$, i. e., $(S, \mathcal{L})$ is a rational conic bundle with the same degree as $(X, L)$. In case b) we have $L^{2}=4 r=\mathcal{L}^{2}$, hence $(X, L)$ and $(S, \mathcal{L})$ have the same degree again. To say more on this case, note that, in particular,

$$
b=2 r .
$$

Let $\mathcal{F}$ be the rank-2 vector bundle on $\mathbb{P}^{1}$, normalized as in [7, p. 373], such that $S=\mathbb{P}(\mathcal{F})$, and let $C_{0}$ and $f$ be the tautological section and a fiber respectively. Then $C_{0}^{2}=-\epsilon$, where $\epsilon=-\operatorname{deg} \mathcal{F}$. Then $\mathcal{L}=\left[r C_{0}+s f\right]$ for some integer $s$ satisfying the ampleness condition $s>r \epsilon$ [7, Corollary 2.18, p. 380]. From the relation

$$
2 b=a_{22}=\rho \mathcal{L}^{2}=\mathcal{L}^{2}
$$

and (31) we can compute the degree of $(S, \mathcal{L})$, getting $4 r=2 b=\mathcal{L}^{2}=r(2 s-r \epsilon)$. This gives $s=2+\frac{r \epsilon}{2}$. Then the parity of $r \epsilon$ and the ampleness condition, combined with the positivity of $r$, lead to a short list of possibilities for $(\epsilon, r, s)$ : namely, either $(0, r, 2)$ with $r$ any positive integer satisfying $r=\frac{L^{2}}{4}$, or $(1,2,3)$, or $(2,1,3)$. In the former case $(S, \mathcal{L})$ is again a rational conic bundle with respect to the second projection; in the second case it is also a conic bundle since $r=2$. Finally, in 
the latter case we have $(S, \mathcal{L})=\left(\mathbb{F}_{2},\left[C_{0}+3 f\right]\right)$ (which is not a conic bundle), and $L^{2}=\mathcal{L}^{2}=4$. So $(X, L)$ itself would be a conic bundle of degree 4 , and it is immediate to check that the only such surface is $\left(\mathbb{P}^{1} \times \mathbb{P}^{1}, \mathcal{O}(2,1)\right)$, up to exchanging the rulings (case (i) in Proposition 2).

In conclusion, what we proved is the following result.

Proposition 12. Let $(X, L)$ be a conic bundle of degree d over a smooth projective curve of genus $q$, with $(X, L) \neq\left(\mathbb{P}^{1} \times \mathbb{P}^{1}, \mathcal{O}(2,1)\right)$, let $\Gamma$ be its Hilbert curve, and suppose that $(S, \mathcal{L})$ is any other polarized surface having $\Gamma$ as Hilbert curve. Then $(S, \mathcal{L})$ is also a conic bundle of degree $d^{\prime}$ over a smooth curve of genus $q^{\prime} ;$ moreover,

(1) $\left(d^{\prime}, q^{\prime}-1\right)$ is proportional to $(d, q-1)$ if $q \geq 2$;

(2) $q^{\prime}=1$ (and $d^{\prime}$ is arbitrary) if $q=1$;

(3) $\left(d^{\prime}, q^{\prime}\right)=(d, 0)$ if $q=0$.

On the other hand, if $(X, L)=\left(\mathbb{P}^{1} \times \mathbb{P}^{1}, \mathcal{O}(2,1)\right)$, then its Hilbert curve is the same as that of the scroll $\left(\mathbb{F}_{2},\left[C_{0}+3 f\right]\right)$.

\section{REFERENCES}

[1] A. Beauville, Complex Algebraic Surfaces, 2nd Edition, Cambridge University Press, 1996 (translation of Surfaces algébriques complexes, Astérisque, 54 Soc. Math. France, Paris (1978)).

[2] M. C. Beltrametti, A. J. Sommese, The Adjunction Theory of Complex Projective Varieties, de Gruyter Expositions in Math., Vol. 16, de Gruyter, 1995.

[3] M. C. Beltrametti, A. Lanteri, and A. J. Sommese, Hilbert curves of polarized varieties, J. Pure Appl. Algebra 214 (2010), 461-479.

[4] F. Campana, Remarques sur le revêtement universel des variétés kählériennes compactes, Bull. Soc. Math. France 122 (1994), no. 2, 255-284.

[5] T. Fujita Classification of polarized manifolds of sectional genus two, in "Algebraic Geometry and Commutative Algebra", I, in honour of M. Nagata. Kinokuniya, Tokyo, 1987, pp. $73-98$.

[6] T. Fujita, Classification Theories of Polarized Varieties, London Math. Soc. Lecture Notes Series, Vol. 155, Cambridge Univ. Press, 1990.

[7] R. Hartshorne, Algebraic Geometry, Graduate Texts in Mathematics, vol. 52, Springer, New York, 1977.

[8] A. Lanteri, Scrolls over surfaces allowing quadric bundle structures over curves are the expected ones. Istit. Lombardo Accad. Sci. Lett. Rend. A 132 (1998), no. 1-2, 3-13 (2000).

[9] A. Lanteri, Characterizing scrolls via the Hilbert curve, Internat. J. Math. 25 (2014), no. 11, 1450101, $17 \mathrm{pp}$.

[10] A. Lanteri, M. Palleschi, and A. J. Sommese, Del Pezzo surfaces as hyperplane sections. J. Math. Soc. Japan 49 (1997), no. 3, 501-529.

Dipartimento di Matematica "F. Enriques", Università degli Studi di Milano, Via C. Saldini, 50, I-20133 Milano, Italy

E-mail address: antonio.lanteri@unimi.it 